\title{
On the role of the gas environment, electron-dose-rate, and sample on the image resolution in transmission electron microscopy
}

\author{
Martin Ek ${ }^{1}$ D, Sebastian P. F. Jespersen ${ }^{1,2}$, Christian D. Damsgaard ${ }^{2}$ and Stig Helveg ${ }^{1 *}$
}

\begin{abstract}
The introduction of gaseous atmospheres in transmission electron microscopy offers the possibility of studying materials in situ under chemically relevant environments. The presence of a gas environment can degrade the resolution. Surprisingly, this phenomenon has been shown to depend on the electron-dose-rate. In this article, we demonstrate that both the total and areal electron-dose-rates work as descriptors for the dose-rate-dependent resolution and are related through the illumination area. Furthermore, the resolution degradation was observed to occur gradually over time after initializing the illumination of the sample and gas by the electron beam. The resolution was also observed to be sensitive to the electrical conductivity of the sample. These observations can be explained by a charge buildup over the electron-illuminated sample area, caused by the beam-gas-sample interaction, and by a subsequent sample motion induced by electrical capacitance in the sample.
\end{abstract}

Keywords: Transmission electron microscopy, TEM, Resolution, Dose-rate, In situ, Gas environment, Sample charging

\section{Background}

The understanding of chemical processes at solid surfaces should benefit from observations made at the atomic scale. While transmission electron microscopy (TEM) has become a prime technique for visualizing matter at atomic resolution, such investigations have mainly been conducted under high vacuum conditions. High-resolution TEM capabilities have recently been extended by the introduction of differentially pumped vacuum systems [1-6] and closed, electron-transparent cells [7-11] that allow relevant gas environments to be confined to the specimen region. Hereby, solid materials can be immersed in chemically relevant environments, while the number of atoms traversed by the electron beam is reduced sufficiently to enable observations in situ by means of TEM.

With this instrumentation, a vast number of reports have unveiled new important information about atomic dynamics at gas-solid interfaces, as reviewed in, e.g.,

\footnotetext{
*Correspondence: sth@topsoe.dk

${ }^{1}$ Haldor Topsoe A/S, Haldor Topsøes Allé 1, 2800 Kgs Lyngby, Denmark Full list of author information is available at the end of the article
}

references [12-15]. In contrast, only a few studies have addressed the physical mechanisms by which the additional electron scattering by the gas environment contributes to the image formation. This scattering has experimentally been shown to depend on the extension, atomic density, and elemental composition of the gas environment, and has been suggested to be both elastic and inelastic in origin [5,16-21]. As a consequence, the electron beam is broadened during its passage through the gas environment, and the resulting image signal is reduced correspondingly. Moreover, calculations suggest that the increased spread of illumination angle and energy will further act by blurring the image and offsetting the image signal at higher magnifications [18, 21, 22]. Overall, these effects indicate that the highest achievable resolution should be signal dependent and thus sensitive to the accumulated electron dose.

A few studies have addressed experimentally the achievable image resolution for solids immersed in gas environments and confirmed the signal-dependent resolution $[5,9,17,18]$. Surprisingly, it has become clear that the signal loss cannot boundlessly be compensated for by only increasing the electron accumulation during 
image acquisition. Studies reporting measurements of the image resolution as a function of gas pressure have demonstrated a dependency on either the areal (Jinschek and Helveg [5]) or total (Bright et al. [16], and more recently Wagner and Beleggia [17]) electron-dose-rate. Thus, the apparent image resolution depends on the way in which the electrons are delivered in addition to the number of electrons accumulated on the detector. As a hypothesis, the dose-rate dependency of the resolution was attributed to inelastic interactions between the primary electrons and gas molecules $[5,16]$. This proposal is consistent with the observation of a more pronounced dose-rate dependency for $\mathrm{N}_{2}$ compared to $\mathrm{H}_{2}$, and for a primary electron energy of 80 compared to $300 \mathrm{keV}$, corresponding to higher inelastic electron-scattering crosssections [5]. Jinschek and Helveg additionally speculated that a local charging of the illuminated specimen area could be causing motion of the specimen through a capacitive effect, whereby smearing of the smallest image features leads to degradation of the resolution. As the degree of charging of the specimen depends on its electrical conductivity, this parameter is expected to also play a role for the achievable resolution.

In this contribution, we will examine the dose-rate dependency of the achievable TEM resolution of a solid specimen immersed in a gas environment in further detail. First, we address the relationship between the previously reported areal and total dose-rate dependencies of the resolution and establish a coherent description of the phenomena. Second, we show that the resolution decays over time at high electron-dose-rates and that the resolution furthermore improves for a specimen with an increased electrical conductivity. These findings give experimental support for the proposal by Jinschek and Helveg [5] that charge accumulated on the sample as a result of the beam-gas-sample interactions represent a cause for the dose-rate dependency of the image resolution.

\section{Methods}

The image resolution limits were examined using a FEI Titan 80-300 ETEM operated with a primary electron energy of $300 \mathrm{keV}$ and with 5 mbar $\mathrm{N}_{2}$ added to the sample region. At these conditions, an electron-dose-ratedependent resolution degradation was reported in a previous study [5]. The total dose-rate (beam current) and the areal dose-rate (beam current density) are connected via the area of the illumination. A change in total doserate can be accomplished by changing the illumination area at a fixed areal dose-rate, which in practice is most easily done by changing the condenser aperture. The total dose-rate can also be varied by changing the areal doserate for a fixed illumination area, which can be done by changing the first condenser lens, or second gun anode, and compensating for any change in beam size with the lower condenser lenses. Likewise, the areal dose-rate can be changed either by changing the total dose-rate for a fixed illumination area, or by changing the illumination area at a fixed total dose-rate by spreading or contracting the electron beam using the lower condenser lenses. Here, the variation in total dose-rate was accomplished by changing the second condenser aperture, which kept the areal dose-rate constant. Variation in areal dose-rate was accomplished by changing the second and third condenser lenses to spread out or contract the electron beam on the sample at a constant total dose-rate.

At each setting, the total and areal electron-dose-rates and illumination diameters were measured in vacuum with no sample in the field-of-view from images acquired at a lower magnification, such that the entire electron beam was included on the charge-coupled device (CCD) camera (Gatan US1000). The summed intensity from the whole image was used to calculate the total doserate, and the average pixel intensity in the central ca. $50 \times 50 \mathrm{~nm}^{2}$ area was used to calculate the areal doserate. A factory-provided conversion factor of 3.8 counts/ $\mathrm{e}^{-}$was used to convert the intensities to the dose-rates. The dose-rates were in this way consistently noted for the electron beam incident on the gas environment and sample, and therefore excluded any signal attenuation due to electron scattering by the gas and sample. To aid in the comparison with previous reports, it can be noted that the total dose-rates reported here of ca. $2-20 \times 10^{9} \mathrm{e}^{-} / \mathrm{s}$ correspond to $0.3-3 \mathrm{nA}$, and that the areal dose-rates of ca. $500-5000 \mathrm{e}^{-} /\left(\AA^{2} \mathrm{~s}\right)$ correspond to $0.8-8 \mathrm{~A} / \mathrm{cm}^{2}$. This covers approximately the same range of dose-rates studied by Jinschek and Helveg [5] and Bright et al. [16].

For Figs. 1, 3, and 4, single exposure images were acquired using the pre-specimen beam blanker to define the image acquisition times. This means that the sample and gas was only illuminated by the electron beam for the duration of the acquisition time. These times were varied to compensate both for the variations in the areal dose-rate and the signal attenuation due to electron scattering by the gas, so that all images were acquired with the same number of electrons collected on the CCD camera. The reference point was set to a $1 \mathrm{~s}$ acquisition at the medium areal dose-rate in vacuum. The required acquisition times varied between $0.25 \mathrm{~s}$ for the highest areal dose-rate in vacuum to $6 \mathrm{~s}$ for the lowest areal dose-rate in 5 mbar $\mathrm{N}_{2}$. This strategy was adopted to avoid signal limitations on the measured resolution in any image [5]. At shorter acquisition times, the image resolution was similar or reduced, which rules out that the resolution reduction at longer acquisition times was caused by sample or defocus drifts. The high-resolution TEM images 
were acquired from $49 \times 49 \mathrm{~nm}^{2}$ areas with a sampling of $0.024 \mathrm{~nm} /$ pixel. These images were used to calculate the Fast Fourier Transforms (FFTs) in each figure and are represented by cropped displays in Additional file 1: Fig. S1 and Fig. S2, respectively. The FFTs are displayed with the same contrast limits. For example, a low limit and a high limit of 0 and $1.5 \times 10^{5}$ counts, respectively, are used for Fig. 1. Typically small variations below $10 \%$ in the total collected signal remained after the compensation by the exposure times, and the high contrast limit for a series of FFTs was adjusted accordingly. This aids in the visual comparison of the FFTs, and has no effect on the measured resolution.

For Fig. 2, series of images were recorded back-toback in rapid succession with $0.2 \mathrm{~s}$ acquisition times in order to monitor the image resolution as a function of time. The image series were acquired from $49 \times 49 \mathrm{~nm}^{2}$ areas using a reduced sampling of $0.048 \mathrm{~nm} /$ pixel, resulting in a recording rate of approximately 1 frame per second. The FFT of each frame in the series was radially unwrapped, background subtracted, and finally averaged to produce the radial profiles shown in Fig. 2. The details of the image processing are given in Additional file 1: Fig. S3.

The image resolution was measured from two samples that differ in mechanical and electrical properties. The first sample consisted of a standard copper grid covered with an amorphous carbon film pre-loaded with goldpalladium nanoparticles (Agar S106). This type of sample was also used for the studies by Jinschek and Helveg [5] and Bright et al. [16] and therefore facilities a comparison with the present examination. This sample is hereafter referred to as the gold-on-carbon sample as the palladium content can be neglected for the purpose of this study. The second sample was a microelectromechanical system (MEMS) device (Protochips Aduro [23]). The MEMS device contains a partially crystalline $\mathrm{SiC}$ film. As the $\mathrm{SiC}$ film can be resistively heated, its temperature-dependent properties can be systematically changed, and their influences on image resolution be addressed while maintaining an identical structure and composition of the sample. The image resolution was measured at 40 or $400{ }^{\circ} \mathrm{C}$ in the presence of $5 \mathrm{mbar}_{2}$. The elevated temperatures were obtained by passing an electrical current through the film. The manufacturer provided a temperature calibration obtained under high vacuum conditions. To compensate for the increased heat transfer by a surrounding gas atmosphere, the heater current was increased to maintain the $\mathrm{SiC}$ film resistance equal to the value corresponding to the manufacturer's vacuum calibration for a given temperature [24].

For both samples, the image resolution was defined as the highest spatial frequency lattice fringe present in a corresponding high-resolution TEM image. This "fringe resolution" is known to be an imperfect measure of resolution in TEM [25], but remains useful for discerning trends in the underlying "true" resolution.

\section{Results and discussion}

Figure 1 shows FFTs of images acquired from the goldon-carbon specimen in 5 mbar $\mathrm{N}_{2}$ as a function of the total and areal electron-dose-rates. The achieved image resolution is estimated from the highest spatial frequency lattice fringe transferred to the image, as indicated by the superimposed dashed circles. As a function of the total dose-rate, the image resolution was close to the inherent information limit of the microscope at the lowest value and decreased at higher values, as previously reported by Bright et al. [16]. On the contrary, a variation of the areal dose-rate at a constant total dose-rate had negligible effect on the resolution. Thus, the resolution did not depend on how the electrons were distributed across the sample. This is particularly clear at the lowest total doserate, where the intrinsic resolution was maintained for all areal dose-rates. At the intermediate total dose-rate $\left(7.5 \times 10^{9} \mathrm{e}^{-} / \mathrm{s}\right)$, the 220 reflection did disappear at the lowest areal dose-rate, which could indicate a resolution reduction from the 220 reflection, corresponding to a lattice spacing of $1.4 \AA$, to the 200 reflection, corresponding to a lattice spacing of $2.0 \AA$. However, the intensity of the 220 reflection was low in all FFTs at the intermediate total dose-rate. Its presence was therefore highly sensitive to sample drift during longer exposure times or reduced signal at shorter acquisition times for the lowest areal dose-rate. The absence of the 220 in this case is therefore more indicative of the sample stability and signal limitation, rather than direct beam-gas-sample interactions. At the highest total dose-rate, the crystal lattice reflections were all absent.

At first glance, this finding seems to contradict the observations of Jinschek and Helveg [5], who demonstrated a clear improvement of the resolution at reduced areal dose-rates. However, it is important to distinguish whether the reduction in areal dose-rate is accomplished at a constant total dose-rate or at a constant illumination area. In the diagram in Fig. 1, varying the dose-rate diagonally corresponds to keeping the illumination area roughly constant as indicated by the disk superimposed in each FFT. Varying the areal dose-rate along the diagonal will clearly result in an improvement of the achievable resolution, and this was the method employed by Jinschek and Helveg (personal communication). In fact, as a reduced areal dose-rate at a constant illumination area also implies a reduced total dose-rate, the two studies are actually in agreement and describe the same phenomenon. 


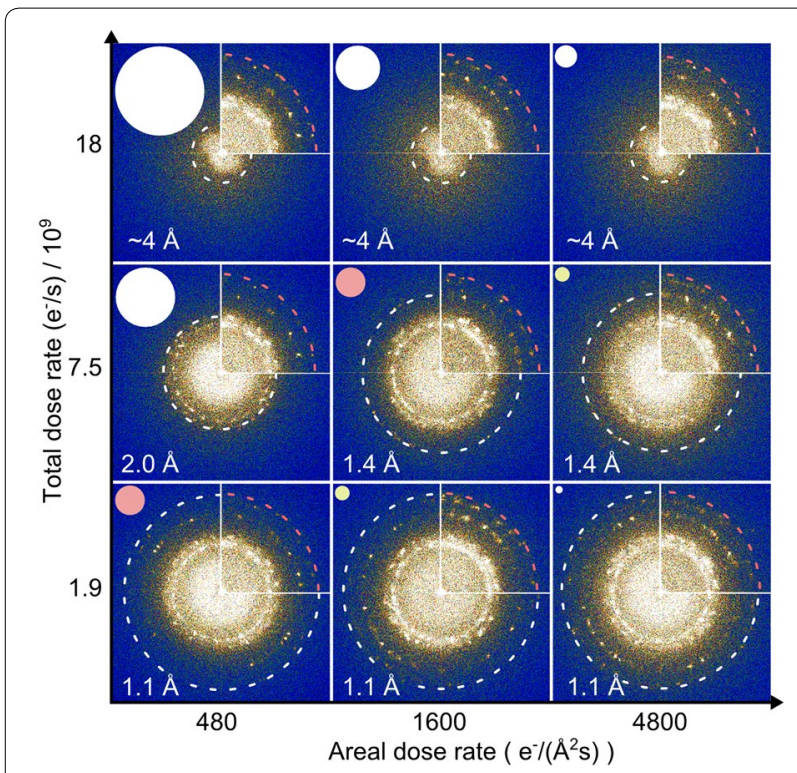

Fig. 1 Image resolution for a gold-on-carbon sample in 5 mbar $\mathrm{N}_{2}$ as a function of total and areal electron-dose-rates. The displays are FFTs of high-resolution TEM images (Additional file 1: Fig. S1). The image resolution is denoted for each illumination condition and corresponds (as indicated by a dashed circle) to the lattice fringe with the highest spatial frequency, except at the highest total dose-rate at which the resolution represents an estimate from the onset of the sudden drop in intensity in the FFTs. The inset in the upper right corner of each FFT shows the corresponding result from an image acquired in vacuum. In each FFT, the disk denotes the relative size of the illuminated sample area and varied from 50 to $600 \mathrm{~nm}$ in diameter. Disks colored yellow and red corresponds to identical diameters of 100 and $200 \mathrm{~nm}$, respectively

Although the total dose-rate and areal dose-rate at a constant illumination area are equally valid descriptors for the TEM image resolution of specimens immersed in a gas environment, the latter has significant advantages. For example, while contracting the beam at a constant total dose-rate has no effect on the resolution, it will increase the extent of beam-induced changes to the sample as these are reported to be determined primarily by the areal dose-rate [26-30]. Conversely, expanding the electron beam puts surrounding areas at risk for unintentional exposure and structural alterations. Such changes may not always be visible immediately, but can have long term consequences for the dynamic behavior of the sample in the gas environment [31]. Observations from liquid cell in situ TEM furthermore show that ionized species formed outside the imaged area when using an overly expanded illumination can have large effects on the observed reactions [32,33]. For these reasons, we suggest that the best practice will be to always report the areal dose-rate and illumination area and keeping the latter as closely matched to the imaged area as possible.

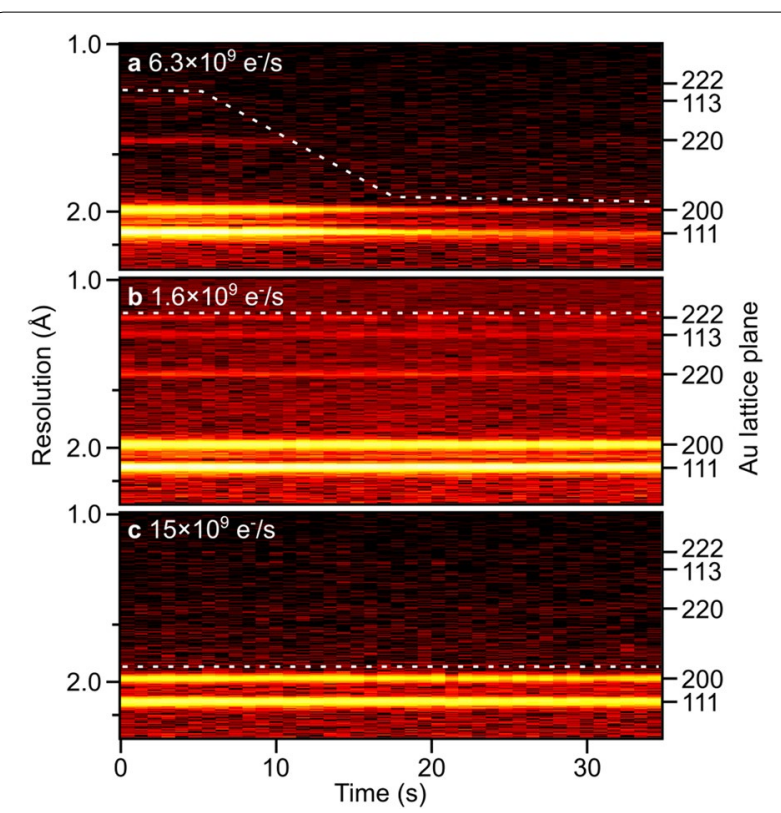

Fig. 2 Time-dependent resolution during prolonged electron illumination. Radial profiles of FFTs from a series of images recorded backto-back, after unblanking the electron beam at time $0 \mathrm{~s}$, with $0.2 \mathrm{~s}$ acquisition times in 5 mbar $\mathrm{N}_{2}$. The image series were acquired from the same area on the gold-on-carbon sample with a near-continuous illumination at three different total dose-rates in the chronological order, a $\left(6.3 \times 10^{9} \mathrm{e}^{-/ \mathrm{s}}\right), \mathbf{b}\left(1.6 \times 10^{9} \mathrm{e}^{-/ \mathrm{s}}\right)$ and $\mathbf{c}\left(15 \times 10^{9} \mathrm{e}^{-/ \mathrm{s}}\right)$. An areal dose-rate of $5600 \mathrm{e}^{-} /\left(\AA^{2} \mathrm{~s}\right)$ was used for all three series. The dashed lines outline the smallest lattice spacing in the images

The images for Fig. 1 were recorded using the prespecimen beam blanker, which deflects the electron beam well above the sample and gas and thereby limits the interaction of the electron beam with the specimen and gas to only the time used for the actual image acquisition. This procedure proved beneficial because the resolution degraded over time when the sample was illuminated with the electron beam. Figure 2 shows this temporal development of the resolution using radial profiles of FFTs calculated from series of images recorded back-to-back in rapid succession with short acquisition times $(0.2 \mathrm{~s})$. The first series was recorded at an intermediate total dose-rate (Fig. 2a) and showed a noticeable decrease in the resolution from the initial $1.2 \AA$ to the final $2.0 \AA$ over approximately $20 \mathrm{~s}$. This gradual reduction in resolution is decidedly not due to accumulation of beam-induced changes at the imaged area because the subsequent series (Fig. 2b), recorded from the very same area at a low total dose-rate, showed that the resolution had recovered to a time-independent value of ca. 1.1 $\AA$. At the highest total dose-rate, the resolution was reduced to $2.0 \AA$ already within the first frame after un-blanking the electron beam (Fig. 2c). This finding indicates that the resolution decayed much 


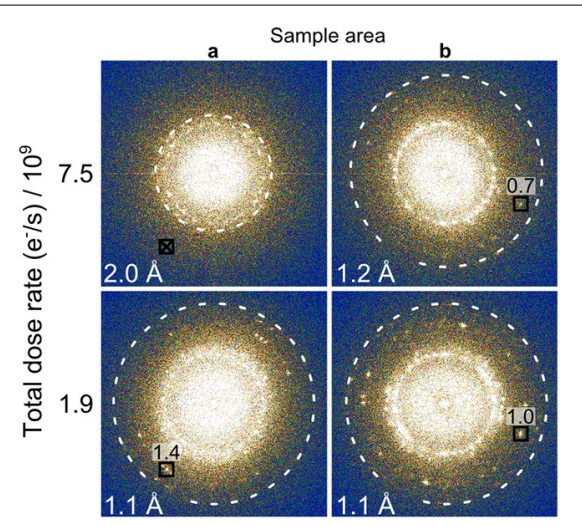

Fig. 3 Location dependency of the image resolution. The displays show FFTs of images recorded from two different areas of the gold-on-carbon sample in $5 \mathrm{mbar}_{2}$ using a low areal dose-rate of $480 \mathrm{e}^{-} /\left(\AA^{2} \mathrm{~s}\right)$. The images from area a are the same as used in Fig. 1, and the images from $\mathbf{b}$ were recorded from a distant area. The image resolution is denoted for each display and corresponds to the highest spatial frequency lattice fringes (indicated by a dashed circle). While the measured resolution at the low total dose-rate was equivalent for the two areas, the reduction in resolution at the higher total doserate was markedly smaller at area $\mathbf{b}$ than at area $\mathbf{a}$. The contrast for a set of strong 220 lattice fringes (black box) is given as the intensity of the corresponding spot in each FFT relative to the intensity of such fringes in area $\mathbf{b}$ at low total dose-rate. The relative higher 220 intensity in $\mathbf{a}$ than in $\mathbf{b}$ at the low total dose-rate indicates that its disappearance at the higher dose-rate is not due to a signal limitation caused by the properties of the gold particles

faster at the highest total dose-rate than at the lower total dose-rates.

In view of these observations, the advantage of using the intermittent acquisition strategy described for Fig. 1 can be attributed to the suppression of the gradual resolution degradation that would have occurred under a prolonged illumination by the electron beam. This is illustrated by the intermediate total dose-rate series in Fig. 2a: a prolonged illumination by the electron beam prior to the acquisition would in this case rapidly have limited the resolution to $2.0 \AA$, rather than the $1.2 \AA$ present in the initial frames. It should be noted that the resolution degradation at high total dose-rates cannot boundlessly be mitigated by this acquisition strategy. Acquisition times below $0.2 \mathrm{~s}$ would, for example, be required to preempt the resolution decay at the highest total dose-rates reported here (see Fig. 2c). Such short exposure times will inevitably lead to limitations due to a low signal-to-noise ratio instead.

The fact that the resolution degradation occurred gradually over tens of seconds (Fig. 2a) indicates that it is related to a dynamic response of the sample under the electron illumination. This response is influenced by the gas environment, because the resolution was unaffected by both the total and areal dose-rates in vacuum, as

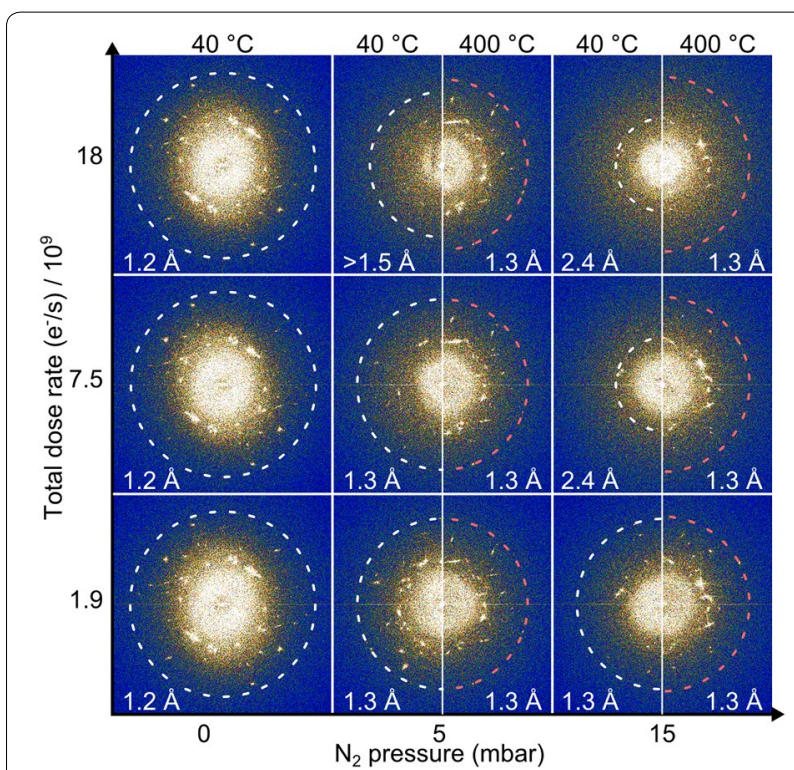

Fig. 4 Image resolution for a $\mathrm{SiC}$ film as a function of the total doserate, $\mathrm{N}_{2}$ pressure and temperature. The displays show FFTs of highresolution TEM images of the partially crystalline SiC film (Additional file 1: Fig. S2). The image resolution is denoted for each condition and corresponds to the lattice fringes with the highest spatial frequency (as indicted by a dashed circle). Images were acquired at two different temperatures of 40 and $400{ }^{\circ} \mathrm{C}$. At 5 and $15 \mathrm{mbar}_{2}$, the displays are cropped to show the left and right half of the FFTs of the full images acquired at 40 and $400{ }^{\circ} \mathrm{C}$, respectively. The illumination diameter was fixed as $100 \mathrm{~nm}$ for the lowest total dose-rate, resulting in a constant areal dose-rate of $1600 \mathrm{e}^{-} /\left(\AA^{2} \mathrm{~s}\right)$. The same areal dose-rate was used also for the higher total dose-rates

shown in Fig. 1. However, the solid sample must also be involved in the resolution degradation, because marked variations were observed between different areas on the gold-on-carbon sample. For instance, the maximum attainable resolution varied between $1.2-2.0 \AA$ at the intermediate total dose-rate (Fig. 3) and 2.0-4 $\AA$ at the highest total dose-rate. Although the absolute values of the resolution varied between different areas, the trends shown in Fig. 1 were commonly found within each individual area. Similarly, the rate at which the resolution degraded over time also varied from area to area compared to the example given in Fig. 2, as illustrated by Additional file 1: Fig. S4. The variability between different areas was not due to differences in image signal caused by differences in the size, orientation, or stability of the deposited gold particles. This is evident from the observation that the intrinsic resolution of the microscope was consistently achieved and maintained over long periods of time at the lowest total dose-rate, as exemplified for one additional area in Fig. 3. The variability between different areas on the sample for identical gas and illumination conditions therefore indicates that the resolution 
reduction depends on local properties of the sample, for example, its propensity for charging under the electron beam. Charges accumulated locally in the sample could be an important factor in the resolution degradation as argued by Jinschek and Helveg [5]. In comparison, recent TEM observations made in situ using a liquid cell showed that sample charging can induce movements in nanoparticles [34]. The amount of charge buildup in the illuminated sample area will depend on the impingement rate of primary electrons, the secondary electron yield from the specimen upon impact, and the local electrical conductivity of the specimen $[35,36]$. The sample can subsequently interact either through charge exchange or electrostatics with the surrounding free space charge formed due to the ionizability of the gas molecules, which would explain the role of the gas phase on the time-dependent resolution. The ionization of the gas molecules can be caused both by primary electrons entering the specimen area and by secondary electrons ejected from the specimen. However, in TEM, the secondary electron yield is typically low (of the order of 1 secondary electron per 100 primary electrons) [36]. Furthermore, the majority of the secondary electrons produced will have too low energy to ionize gas molecules [36]. Therefore, it is likely that the secondary electrons mainly affect sample charging, and that it is the primary electrons which dominate gas ionization.

The role of the sample material was addressed by an examination of the SiC MEMS sample. Figure 4 shows FFTs of TEM images of the SiC film and illustrates the effect of the total dose-rate on the resolution under vacuum $\left(10^{-6} \mathrm{mbar}\right), 5$ and $15 \mathrm{mbar}_{2}$. In vacuum, a lattice fringe resolution of $1.2-1.3 \AA$ was typically achieved. The apparent lower resolution measured from the $\mathrm{SiC}$ film compared to the gold-on-carbon sample is attributed to the lower electron-scattering power of $\mathrm{SiC}$ that reduces the contrast for all lattice fringes and thereby the measured resolution. For the $\mathrm{SiC}$ film, a measured resolution of $1.2-1.3 \AA$ is therefore taken as an indication that the intrinsic resolution of the microscope was maintained. Thus, even if the absolute values differ slightly, the trends in resolution with electron-dose-rate can still be compared for the two sample types (Figs. 1, 4).

As shown by the two first columns at $40{ }^{\circ} \mathrm{C}$ in Fig. 4, increasing the total dose-rate had no measurable effect on the measured resolution in vacuum but led to a resolution degradation in 5 mbar $\mathrm{N}_{2}$ similar to the gold-oncarbon sample. The $\mathrm{SiC}$ film, however, appeared to be less sensitive to the total dose-rate than the gold-on-carbon sample, as the resolution at 5 mbar $\mathrm{N}_{2}$ was retained at the intermediate total dose-rate $\left(7.5 \times 10^{9} \mathrm{e}^{-} / \mathrm{s}\right)$ and remained better than $2 \AA$ at the highest total dose-rate $\left(18 \times 10^{9} \mathrm{e}^{-} / \mathrm{s}\right)$. At $15 \mathrm{mbar}_{2}\left(40^{\circ} \mathrm{C}\right)$, the intrinsic resolution for the SiC film could be maintained only for the lowest total dose-rate, but even at this pressure $2.4 \AA$ fringes remained at the highest total dose-rate. These observations demonstrate that the resolution was maintained for the $\mathrm{SiC}$ sample to higher total dose-rates than for the gold-on-carbon sample under the same experimental conditions, and are thus further evidence that the sample plays a crucial role.

The specific reasons for the increased tolerance to high total dose-rates for the $\mathrm{SiC}$ sample are difficult to discern. Possibilities include a higher electrical conductivity from the illuminated area to the specimen holder, a reduced secondary electron yield, or a higher degree of mechanical stability. The SiC film in the MEMS device can be resistively heated [23], which offers a way to systematically change its properties and examine the corresponding resolution, while otherwise maintaining an identical sample. The effect of heating the $\mathrm{SiC}$ film is also illustrated by Fig. 4, which shows FFTs calculated from images recorded at 40 and $400{ }^{\circ} \mathrm{C}$. The FFTs show that, for both 5 and 15 mbar $\mathrm{N}_{2}$, the resolution was better at $400{ }^{\circ} \mathrm{C}$ than at $40{ }^{\circ} \mathrm{C}$ for the higher total dose-rates, while the resolution was the same at the lowest total doserate. In fact, the intrinsic resolution could be retained at $400{ }^{\circ} \mathrm{C}$ even at the highest total dose-rate. At 5 mbar and $400{ }^{\circ} \mathrm{C}$, the highest total dose-rate did not even result in a reduced contrast for the highest spatial frequency lattice fringes as indicated by the intensity of the outermost reflection in the FFT. A small reduction in contrast occurred at 15 mbar, resulting in the loss of some lattice fringes without affecting the measured resolution.

To interpret the observations in Fig. 4, it is important to consider that upon heating the $\mathrm{SiC}$ film, the surrounding gas will also be locally heated, which will reduce its atom density. For an ideal gas, a temperature increase from 40 to $400{ }^{\circ} \mathrm{C}$ will roughly halve its molar volume. As the dose-rate-dependent resolution depends on the gas pressure (see Fig. 4), any effect of such a reduced gas density must be addressed as well. A local expansion of the gas closest to the sample can, however, be counteracted by increasing the overall pressure in the microscope. Specifically, the atom density for an ideal gas at $15 \mathrm{mbar}$ and $400{ }^{\circ} \mathrm{C}\left(1.6 \cdot 10^{17}\right.$ molecules $\left./ \mathrm{cm}^{3}\right)$ is slightly higher than at 5 mbar and $40{ }^{\circ} \mathrm{C}\left(1.2 \cdot 10^{17}\right.$ molecules/ $\mathrm{cm}^{3}$ ). Therefore, one might expect that the dose-ratedependent resolution at 15 mbar and $400{ }^{\circ} \mathrm{C}$ should be similar to or lower than at $5 \mathrm{mbar}$ and $40{ }^{\circ} \mathrm{C}$. Instead, Fig. 4 shows that the intrinsic resolution consistently was retained also at $15 \mathrm{mbar}$ for the heated sample. This finding excludes the reduced gas density as an explanation for the reduced sensitivity to the total electron-dose-rate 
for the heated sample. Instead the temperature-dependent properties of the semiconducting $\mathrm{SiC}$ film appear as more obvious candidates. The thermal expansion of $\mathrm{SiC}$ amounts to only ca. $0.1 \%$ for a temperature increase from 40 to $400{ }^{\circ} \mathrm{C}$ (calculated using a linear expansion coefficient of $2.5 \cdot 10^{-6} \mathrm{~K}^{-1}$ [37]) and can therefore be neglected. Sample drift is expected to increase slightly with temperature for the MEMS device and would, if anything, worsen the apparent resolution [38]. In contrast to the thermal expansion and drift, the measured electrical resistivity of the semiconducting $\mathrm{SiC}$ film decreased markedly from 12 to $7 \mathrm{k} \Omega$ upon heating up from 40 to $400{ }^{\circ} \mathrm{C}$. The corresponding increase in electrical conductivity will improve de-charging of the $\mathrm{SiC}$ film and thus lead to a reduced degree of charge buildup at the illuminated area. This provides a clear indication of the crucial role of sample charging on the observed resolution reduction at high electron-dose-rates for samples immersed in gases. The increased temperature may additionally lead to decreased secondary electron yields [39], although this reduction is likely small. For comparison, only a decrease of ca. $10 \%$ in secondary electron yield was reported in the case of $\mathrm{SiO}_{2}$ at $350{ }^{\circ} \mathrm{C}$ compared to room temperature [40].

\section{Conclusions}

The present study indicates that the total dose-rate and areal dose-rate at a fixed illumination area are equivalent descriptors for the achievable TEM image resolution for samples immersed in gas environments. The dose-ratedependent resolution was in previous reports primarily attributed to the combined interaction between the electron beam, gas environment, and sample. The present work gives further support for the role of the sample itself, e.g., by monitoring a temporal evolution of the image resolution while illuminating the sample, and by examining the influence of the electrical conductivity of the sample on the image resolution. These findings indicate that the resolution degradation could be a result of charge buildup on the sample due to beam-gas-sample interactions. Previously, Jinschek and Helveg suggested that charging may lead to resolution degradation by inducing sample movements mediated through a capacitive effect between different parts of the sample. Such a mechanism may account for the observations presented here. In summary, the present work shows that in situ observations by TEM of samples immersed in gas environments must be performed at low dose-rates in order to maintain resolution possibly due to suppression of electric charging in both the gas and the sample. In turn, such electron illumination conditions are advantageous in terms of minimizing electron beam-induced alterations of the chemical reactions under examination.

\section{Additional file}

Additional file 1. Supplementary figures S1-4. Figures S1 and S2 show details from the original high-resolution TEM images used for producing the FFTs displayed in Figs. 1 and 4 in the main text. Figure S3 illustrates the procedure used for producing the radial FFT profiles displayed in Fig. 2 in the main text. Figure $\mathbf{S 4}$ provides an additional example of the time-dependent resolution degradation, illustrating the variability of this behavior from area to area on the sample.

\section{Authors' contributions}

The idea for the investigation was conceived by SH. All authors contributed to the experimental design and interpretation of the results. ME and SPFJ performed the measurements. ME and SH drafted the manuscript which was subsequently revised and approved by all authors. All authors read and approved the final manuscript.

\section{Author details}

${ }^{1}$ Haldor Topsoe A/S, Haldor Topsøes Allé 1, 2800 Kgs Lyngby, Denmark.

${ }^{2}$ Center for Electron Nanoscopy and Department of Physics, Technical University of Denmark, 2800 Kgs Lyngby, Denmark.

\section{Acknowledgements}

The authors thank Sven UIImann for assistance with the in situ TEM experiments and Joerg Jinschek for fruitful discussions. The Innovation Fund Denmark (Grant Cat-C) and the Danish National Research Foundation's Centre for Individual Nanoparticle Functionality (DNRF54) are gratefully acknowledged for financial support.

\section{Competing interests}

The authors declare that they have no competing interests.

Received: 25 February 2016 Accepted: 15 April 2016

Published online: 04 May 2016

\section{References}

1. Boyes, E.D., Gai, P.L.: Environmental high resolution electron microscopy and applications to chemical science. Ultramicroscopy $\mathbf{6 7}, 219-232$ (1997). doi:10.1016/S0304-3991(96)00099-X

2. Sharma, R., Crozier, P.A.: Environmental transmission electron microscopy in nanotechnology. In: Yao, N., Wang, Z.L. (eds.) Handbook of microscopy for nanotechnology, pp. 531-565. Kluwer Academic Publishers, New York (2005)

3. Hansen, P.L., Helveg, S., Datye, A.K.: Atomic-scale imaging of supported metal nanocluster catalysts in the working state. Adv. Catal. 50, 77-95 (2006). doi:10.1016/S0360-0564(06)50002-1

4. Hansen, T.W., Wagner, J.B., Dunin-Borkowski, R.E.: Aberration corrected and monochromated environmental transmission electron microscopy: challenges and prospects for materials science. Mater. Sci. Technol. 26, 1338-1344 (2010). doi:10.1179/026708310X12756557336355

5. Jinschek, J.R., Helveg, S.: Image resolution and sensitivity in an environmental transmission electron microscope. Micron 43, 1156-1168 (2012). doi:10.1016/j.micron.2012.01.006

6. Takeda, S., Kuwauchi, Y., Yoshida, H.: Environmental transmission electron microscopy for catalyst materials using a spherical aberration corrector. Ultramicroscopy 151, 178-190 (2015). doi:10.1016/j.ultramic.2014.11.017

7. Creemer, J.F., Helveg, S., Hoveling, G.H., Ullmann, S., Molenbroek, A.M., Sarro, P.M., Zandbergen, H.W.: Atomic-scale electron microscopy at ambient pressure. Ultramicroscopy 108, 993-998 (2008). doi:10.1016/j. ultramic.2008.04.014

8. Kawasaki, T., Miura, T., Tsutsui, H., Matsutani, T., Tanji, T.: Development of a new closed-type environmental-cell specimen holder with high gas conductivity. Microsc. Microanal. 16, 332-333 (2010). doi:10.1017/ S1431927610056011 
9. Yaguchi, T., Suzuki, M., Watabe, A., Nagakubo, Y., Ueda, K., Kamino, T.: Development of a high temperature-atmospheric pressure environmental cell for high-resolution TEM. J. Electron Microsc. 60, 217-225 (2011). doi:10.1093/jmicro/dfr011

10. Allard, L.F., Overbury, S.H., Bigelow, W.C., Katz, M.B., Nackashi, D.P., Damiano, J.: Novel MEMS-based gas-cell/heating specimen holder provides advanced imaging capabilities for in situ reaction studies. Microsc Microanal. 18, 656-666 (2012). doi:10.1017/S1431927612001249

11. Li, Y., Zakharov, D., Zhao, S., Tappero, R., Jung, U., Elsen, A., Baumann, P., Nuzzo, R.G., Stach, E.A., Frenkel, A.I.: Complex structural dynamics of nanocatalysts revealed in Operando conditions by correlated imaging and spectroscopy probes. Nat. Commun. 6, 7583 (2015). doi:10.1038/ ncomms8583

12. Gai, P.L., Sharma, R., Ross, F.M.: Environmental (S)TEM studies of gas-liquid-solid interactions under reaction conditions. MRS Bull. 33, 107-114 (2008). doi:10.1557/mrs2008.23

13. Jinschek, J.R.: Advances in the environmental transmission electron microscope (ETEM) for nanoscale in situ studies of gas-solid interactions. Chem. Commun. 50, 2696-2706 (2014). doi:10.1039/c3cc49092k

14. Helveg, S:: An industrial perspective of the impact of Haldor Topsøe on (in situ) electron microscopy in catalysis. J. Catal. 328, 102-110 (2015). doi:10.1016/j.jcat.2014.12.017

15. Crozier, P.A., Hansen, T.W.: In situ and operando transmission electron microscopy of catalytic materials. MRS Bull. 40, 38-45 (2015). doi:10.1557/ mrs.2014.304

16. Bright, A.N., Yoshida, K., Tanaka, N.: Influence of total beam current on HRTEM image resolution in differentially pumped ETEM with nitrogen gas. Ultramicroscopy 124, 46-51 (2013). doi:10.1016/j. ultramic.2012.08.007

17. Wagner, J.B., Belaggia, M.: Gas-electron interaction in the ETEM. In: Hansen, T.W., Wagner, J.B. (eds.) Controlled atmosphere transmission electron microscopy: principles and practice, pp. 63-94. Springer International Publishing, Cham (2016)

18. Suzuki, M., Yaguchi, T., Zhang, X.F.: High-resolution environmental transmission electron microscopy: modeling and experimental verification. Microscopy 62, 437-450 (2013). doi:10.1093/jmicro/dft001

19. Allinson, D.L.: Environmental devices in electron microscopy. In: Hayat, M.A. (ed.) Principles and techniques in electron microscopy, biological applications, vol. 5, pp. 62-113. Van Nostrand Reinhold Company, New York (1975)

20. Cosslett, V.E.: The high-voltage electron microscope. Contemp. Phys. 9 , 333-354 (1968)

21. Butler, E.P., Hale, K.F.: In situ studies of gas-solid reactions. In: Glauert, A.M. (ed.) Practical methods in electron microscopy, vol. 9. North-Holland, Amsterdam (1981)

22. Yoshida, H., Takeda, S.: Image formation in a transmission electron microscope equipped with an environmental cell: single-walled carbon nanotubes in source gases. Phys. Rev. B. 72, 195428 (2005)

23. Allard, L.F., Bigelow, W.C., Jose-Yacaman, M., Nackashi, D.P., Damiano, J., Mick, S.E.: A new MEMS-based system for ultra-high-resolution imaging at elevated temperatures. Microsc. Res. Tech. 72(208-215), 1954 (2009). doi:10.1103/PhysRevB.72.195428

24. Hansen, L.P., Johnson, E., Brorson, M., Helveg, S.: Growth Mechanism for Single- and Multi-Layer $\mathrm{MoS}_{2}$ Nanocrystals. J. Phys. Chem. C 118 22768-22773 (2014). doi:10.1021/jp5069279

25. O'Keefe, M.A.: "Resolution" in high-resolution electron microscopy. Ultramicroscopy 47, 282-297 (1992). doi:10.1016/0304-3991(92)90203-V

26. Simonsen, S.B., Chorkendorff, I., Dahl, S., Skoglundh, M., Sehested, J., Helveg, S.: Direct observations of oxygen-induced platinum nanoparticle ripening studied by in situ TEM. J. Am. Chem. Soc. 132, 7968-7975 (2010). doi:10.1021/ja910094r
27. Kuwauchi, Y., Yoshida, H., Akita, T., Haruta, M., Takeda, S.: Intrinsic catalytic structure of gold nanoparticles supported on $\mathrm{TiO}_{2}$. Angew. Chem. Int. Ed. 51, 7729-7733 (2012). doi:10.1002/anie.201201283

28. Jiang, N., Spence, J.C.H.: On the dose-rate threshold of beam damage in TEM. Ultramicroscopy 113,77-82 (2012). doi:10.1016/j. ultramic.2011.11.016

29. Wagner, J.B., Cavalca, F., Damsgaard, C.D., Duchstein, L.D.L., Hansen, T.W.: Exploring the environmental transmission electron microscope. Micron 43, 1169-1175 (2012). doi:10.1016/j.micron.2012.02.008

30. Vendelbo, S.B., Elkjær, C.F., Falsig, H., Puspitasari, I., Dona, P., Mele, L., Morana, B., Nelissen, B.J., van Rijn, R., Creemer, J.F., Kooyman, P.J., Helveg, S.: Visualization of oscillatory behaviour of Pt nanoparticles catalysing CO oxidation. Nat. Mater. 13(9), 884-890 (2014). doi:10.1038/NMAT4033

31. Van Den Berg, R., Elkjaer, C.F., Gommes, C.J., Chorkendorff, I., Sehested, J., De Jongh, P.E., De Jong, K.P., Helveg, S.: Revealing the formation of copper nanoparticles from a homogeneous solid precursor by electron microscopy. J. Am. Chem. Soc. 138, 3433-3442 (2016). doi:10.1021/jacs.5b12800

32. Schneider, N.M., Norton, M.M., Mendel, B.J., Grogan, J.M., Frances, M., Bau, H.H.: Electron-water interactions and implications for liquid cell electron microscopy. J. Phys. Chem. C 118, 22373-22382 (2014). doi:10.1021/ jp507400n

33. Abellan, P., Woehl, T.J., Parent, L.R., Browning, N.D., Evans, J.E., Arslan, I.: Factors influencing quantitative liquid (scanning) transmission electron microscopy. Chem. Commun. 50, 4873 (2014). doi:10.1039/c3cc48479c

34. Woehl, T.J., Prozorov, T.: The mechanisms for nanoparticle surface diffusion and chain self-assembly determined from real-time nanoscale kinetics in liquid. J. Phys. Chem. C 119, 21261-21269 (2015). doi:10.1021/ acs.jpcc.5b07164

35. Cazaux, J.: Correlations between ionization radiation damage and charging effects in transmission electron microscopy. Ultramicroscopy $\mathbf{6 0}$, 411-425 (1995). doi:10.1016/0304-3991(95)00077-1

36. Mildner, S., Beleggia, M., Mierwaldt, D., Hansen, T.W., Wagner, J.B., Yazdi, S., Kasama, T., Ciston, J., Zhu, Y., Jooss, C.: Environmental TEM study of electron beam induced electrochemistry of $\operatorname{Pr}_{0.64} \mathrm{Ca}_{0.36} \mathrm{MnO}_{3}$ catalysts for oxygen evolution. J. Phys. Chem. C 119, 5301-5310 (2015). doi:10.1021/ jp511628c

37. Taylor, A., Jones, R.M.: Silicon Carbide-a high temperature semiconductor. Pergamon Press, Oxford (1960)

38. Helveg, S., Kisielowski, C.F., Jinschek, J.R., Specht, P., Yuan, G., Frei, H.: Observing gas-catalyst dynamics at atomic resolution and single-atom sensitivity. Micron 68, 176-185 (2014). doi:10.1016/j.micron.2014.07.009

39. Sternglass, E.J.: Theory of secondary electron emission by high-speed ions. Phys. Rev. 108, 1-12 (1957). doi:10.1103/PhysRev.108.1

40. Yamano, Y., Kobayashi, S., Michizono, S., Saito, Y.: Secondary electron emission and surface charging evaluation of alumina ceramics and sapphire. IEEE Trans. Dielectr. Electr. Insul. 13, 72-77 (2006). doi:10.1109/ TDEI.2006.1593403

\section{Submit your manuscript to a SpringerOpen ${ }^{\circ}$ journal and benefit from:}

- Convenient online submission

- Rigorous peer review

- Immediate publication on acceptance

- Open access: articles freely available online

- High visibility within the field

- Retaining the copyright to your article

Submit your next manuscript at springeropen.com 